\title{
PREVALENCIA Y FACTORES ASOCIADOS A MACROSOMÍA EN PERÚ, 2013
}

\author{
Antonio José Ledo Alves da Cunha1,a, Manuel Sobrino Toro ${ }^{2, b}$, César Gutiérrez ${ }^{3, c}$, Jorge Alarcón-Villaverde ${ }^{3, b}$
}

\begin{abstract}
RESUMEN
Objetivos. Determinar la prevalencia de macrosomía y factores asociados en Perú, y describir la ocurrencia de complicaciones durante el parto y posparto. Materiales y métodos. Los pesos al nacer de los niños menores de cinco años fueron analizados usando datos de la Encuesta Demográfica y de Salud Familiar (ENDES) 2013, llevada a cabo por el Instituto Nacional de Estadística e Informática. Los niños con un peso mayor a $4000 \mathrm{~g}$ al nacer fueron considerados como macrosómicos. Se empleó un análisis de regresión logística para establecer la asociación independiente de factores sociodemográficos con la macrosomía. Resultados. La muestra estuvo constituida por 6121 niños. La prevalencia de macrosomía fue 5,3\% (intervalo de confianza al 95\%: 4,8 a 5,9). El sexo masculino, un orden de nacimiento mayor, la obesidad materna y una mayor estatura materna estuvieron independientemente asociados con la macrosomía. Los partos por cesárea fueron más frecuentes en niños macrosómicos (43,9\% vs $26,9 \%)$. Las complicaciones durante el parto y posparto fueron frecuentes, pero no estadísticamente asociadas con la macrosomía. Conclusiones. La prevalencia de macrosomía en Perú es relativamente baja comparada con otros países de ingresos bajos o medios. Los factores asociados con la macrosomía fueron principalmente no modificables, con excepción de la obesidad materna. Los niños macrosómicos nacieron más frecuentemente por cesárea. La reducción del peso y la prevención de la obesidad en mujeres en edad fértil en Perú podrían potencialmente reducir la macrosomía y las tasas de cesáreas.
\end{abstract}

Palabras clave: macrosomía; factores sociodemográficos, cesárea. (fuente: DeCS BIREME).

\section{PREVALENCE AND ASSOCIATED FACTORS OF MACROSOMIA IN PERU, 2013}

\begin{abstract}
Objectives. To determine the prevalence of macrosomia and factors associated with it in Peru and to describe the occurrence of complications peri- and postpartum. Materials and Methods. Birth weights of children under the age of 5 years were analyzed using data from the 2013 Demographic and Family Health Survey (ENDES) carried out by the Instituto Nacional de Estadística e Informática. Children with a birth weight higher than $4000 \mathrm{~g}$ were considered macrosomic. A logistic regression analysis was used to establish the independent association of sociodemographic factors with macrosomia. Results. The sample comprised 6121 children. The prevalence of macrosomia was $5.3 \%$ (95\% interval confidence: 4.8-5.9\%). Being male, a higher birth order, maternal obesity, and greater maternal height were independently linked with macrosomia. Caesarean births were more common in macrosomic children than unaffected ones $(43.9 \%$ vs $26.9 \%)$. Complications during birth and postpartum were common but not statistically linked with macrosomia. Conclusions. The prevalence of macrosomia in Peru is relatively low compared to other low-to-middle income countries. The factors associated with macrosomia were mainly unmodifiable, with the exception of maternal obesity. Macrosomic children were more frequently born by caesarean. Weight reduction and the prevention of obesity in women of childbearing age in Peru could potentially reduce macrosomia and caesarean rates.
\end{abstract}

Key words: Fetal Macrosomia , Cesarean Section, Peru (source: MeSH NLM)

\section{INTRODUCCIÓN}

La macrosomía es una condición obstétrica asociada a un incremento del riesgo de mortalidad perinatal y morbilidad neonatal ${ }^{(1-3)}$. Se considera que los fetos anormalmente grandes presentan macrosomía, sin embargo, no hay consenso sobre la definición de esta condición ${ }^{(1,4)}$. En todo caso, la macrosomía puede tener consecuencias a corto y largo plazo para el recién nacido y la madre ${ }^{(5)}$, haciendo de esta condición un

\footnotetext{
Faculdade de Medicina, Federal University of Rio de Janeiro. Rio de Janeiro, Brasil.

Faculdad de Medicina, Universidad de Sevilla. Sevilla, España.

Instituto de Medicina Tropical Daniel A. Carrión, Universidad Nacional Mayor de San Marcos. Lima, Perú.

Doctor en Epidemiología; ${ }^{\mathrm{b}}$ doctor en Medicina; ${ }^{\mathrm{c}}$ magíster en Epidemiología

Recibido: 22/09/2016 Aprobado: 11/01/2017 En línea: 23/03/2017
} 
problema de salud significativo, especialmente en entornos con recursos limitados para hacer frente a las complicaciones asociadas.

De acuerdo con investigaciones llevadas a cabo en diferentes países y entornos, la prevalencia de macrosomía oscila entre el 0,5 a $14,9 \%{ }^{(6)}$ y del 2,8 a $7,2 \%$ en América Latina ${ }^{(7)}$. Por otra parte, la incidencia de macrosomía pareciera ir en aumento, principalmente debido a un incremento de la obesidad y diabetes materna ${ }^{(6)}$. Entre los factores de riesgo para macrosomía se han descrito una mayor edad materna, el aumento de la talla materna, la paridad elevada, la obesidad, la diabetes gestacional y pregestacional, el embarazo prolongado y el sexo masculino del feto ${ }^{(3,8)}$. Aunque algunos factores de riesgo son no modificables, otros pueden serlo ${ }^{(9)}$. La información sobre la prevalencia de macrosomía y los factores asociados a partir de estudios de base poblacional realizados en países de bajos y medianos ingresos es escasa. Esta información podría ayudar a clarificar si la macrosomía es una condición que se produce tanto en poblaciones ricas como pobres. Además, podría proporcionar datos que promuevan la prevención de la macrosomía. El Perú, con sus distintas regiones geográficas y variaciones socioeconómicas, ofrece la oportunidad de estudiar estas cuestiones.

Se analizaron los datos de una encuesta poblacional de alcance nacional para estimar la prevalencia de macrosomía y los factores asociados en Perú. También describimos la ocurrencia de complicaciones en el parto de niños macrosómicos.

\section{MATERIALES Y MÉTODOS}

Los pesos al nacer de los niños menores de 5 años de edad fueron analizados utilizando los datos de la Encuesta Demográfica y de Salud Familiar (ENDES) 2013, conducida por el Instituto Nacional de Estadística e Informática (INEI). Esta encuesta se basa en una muestra aleatoria, bietápica, de representación nacional, de mujeres de 15 a 49 años y niños de 0-5 años. El marco muestral fue preparado a partir de la información proporcionada por el Censo Nacional de Población y Vivienda 2007. Además, el diseño estratificado permite obtener resultados representativos de las zonas urbanas y rurales $(24 \%$ de la población peruana vive en zonas rurales), las regiones naturales (costa, sierra y selva), Lima Metropolitana ( $28 \%$ de la población nacional) y de cada una de las 25 regiones del Perú ${ }^{(10)}$.

En este análisis se incluyeron niños menores de 5 años de edad con registros de peso al nacer. Si una mujer entrevistada tenía más de un niño menor de 5 años de edad, fue incluido solo el menor de sus hijos (nacimiento más reciente). Se excluyeron los casos de hermanos nacidos de embarazo múltiple.
El peso al nacer fue clasificado como bajo peso al nacer (<2500 g), peso normal (2500 a $4000 \mathrm{~g}$ ) o macrosomía $(>4000 \mathrm{~g})$. Como parte de la metodología de las entrevistas para la ENDES, el peso al nacer se obtuvo a partir de las tarjetas de control del niño o por recuerdo de la madre, si no se contaba con la tarjeta al momento de la entrevista.

Se analizaron las siguientes variables: sexo del niño; el orden de nacimiento (primero, segundo, tercer hijo o más); la edad materna (15-24, 25-34 o 35-49 años); la educación materna (sin educación / primaria, secundaria o la educación superior); el índice de masa corporal de la madre (IMC - ni sobrepeso ni obesidad [IMC <25], sobrepeso [IMC $\geq 25$ a $<30$ ] u obesidad [IMC $\geq 30$ ]); la talla materna ( $<1,50 \mathrm{~m}, 1,50 \mathrm{~m}$ a $<1,55 \mathrm{~m}$ o $\geq 1,55 \mathrm{~m})$; región natural (Lima Metropolitana, resto de costa, sierra o selva); zona de residencia (urbana o rural); altitud ( $<2500$ $0 \geq 2500 \mathrm{~m}$ de altitud), y el índice de riqueza. La ENDES clasifica la situación económica de acuerdo con el índice de riqueza, una puntuación que, en última instancia, se basa en las características de la vivienda, el acceso de los hogares a los servicios, propiedad de electrodomésticos y vehículos, tipo de combustible utilizado para cocinar y el hacinamiento ${ }^{(11)}$. La puntuación se divide en quintiles: muy pobres, pobres, medio, ricos y muy ricos. Para este análisis, las categorías medio, ricos y muy ricos se agruparon como "no pobres".

Las variables sociodemográficas se seleccionaron en función de la disponibilidad en las bases de datos. Las variables biológicas se incluyeron en base a los hallazgos de la literatura científica. Además, las complicaciones del parto (parto prolongado, sangrado excesivo, fiebre, convulsiones $u$ otros); complicaciones posparto (hemorragia intensa, pérdida de la conciencia, fiebre, escalofríos, infección de mamas, disuria, secreción vaginal o pérdida involuntaria de orina), y el tipo de parto (vaginal o cesárea) se compararon entre los nacimientos de peso normal y los niños con macrosomía.

Para analizar los factores asociados a macrosomía, solo se incluyeron los niños cuyo peso al nacer fue obtenida de las tarjetas de control de la natalidad. La presencia de macrosomía en comparación al peso normal al nacer se analizó para cada una de las variables sociodemográficas y biológicas.

El análisis estadístico incluyó el cálculo de la prevalencia de macrosomía (porcentaje de bebés con macrosomía sobre toda la muestra) y la construcción de tablas de distribución de frecuencias para la descripción de las variables de estudio y tablas de contingencia. Para el análisis bivariado se utilizó la prueba de chi cuadrado (fue considerado significativo un valor de $p<0,05)$. Se calculó la odds ratio $(\mathrm{OR})$ con intervalos de confianza al 95\% (IC 95\%) como medida de fuerza de asociación. Se elaboró un modelo de regresión logística 
para estudiar la asociación independiente de cada variable con la macrosomía, obteniendo OR ajustada e IC 95\%. Las variables con $p<0,10$ en el análisis bivariado se incluyeron en el modelo de regresión, verificando la ausencia de colinealidad entre las variables independientes del modelo. El análisis incluyó las recomendaciones de ponderación de las encuestas demográficas y de salud ${ }^{(12)}$. Se utilizó el programa estadístico SPSS 21.0.

Los diferentes módulos de la base de datos de la ENDES 2013 están disponibles gratuitamente en la página web del INEI (http://www.inei.gob.pe/bases-de-datos/). Los registros de la base de datos no proporcionan información que permite la identificación de los participantes, lo que garantiza la confidencialidad. Como parte de la metodología de trabajo de campo de la ENDES, el personal del INEI solicitó a los participantes potenciales su consentimiento para su participación voluntaria en el estudio, antes de la recolección de datos.

\section{RESULTADOS}

Del total de niños registrados en la base de datos de la ENDES 2013 fueron incluidos en este estudio 6121, mientras que 2250 fueron excluidos (1237 no eran los hijos de menor edad de la madre, 612 eran hermanos de embarazo múltiple y 401 no tenían datos sobre el peso al nacer).

La prevalencia de macrosomía fue del 5,3\% (IC 95\% 4,8 a 5,9). Las características de la muestra se presentan en la Tabla 1. El peso al nacer se obtuvo de las tarjetas de control en el $45 \%$ de los niños.

La macrosomía ocurrió con más frecuencia en bajas altitudes y en Lima Metropolitana (Tabla 2). El análisis bivariado reveló que la educación materna $(p=0,08)$ fue la única variable no asociada con la macrosomía (Tabla 2). Puesto que todas las variables presentaron un valor $p<0,10$ en el análisis bivariado, se incluyeron en el modelo multivariado.

El sexo masculino (OR 0,28; IC 95\%:0,18-0,42), tener un orden de nacimiento segundo (OR 2,18; IC 95\%: 1,19-2,40) o de tercero a más (OR 5,38; IC 95\%: $(2,85-10,16)$, la obesidad materna (OR 2,08; IC 95\%: 1,22-3,54) y el aumento de la talla materna, se asociaron de forma independiente con la macrosomía (Tabla 3).

Los niños macrosómicos nacieron en mayor frecuencia por cesárea (OR 2,13; IC 95: 1,50-3,04). Sin embargo, las complicaciones durante el parto y posparto, aunque ambos frecuentes, no se asociaron con la macrosomía (Tabla 4).
Tabla 1. Características de la muestra estudiada

\begin{tabular}{|c|c|c|}
\hline Variable & $n=6121$ & $(\%)$ \\
\hline \multicolumn{3}{|l|}{ Sexo } \\
\hline Masculino & 3141 & $(51,3)$ \\
\hline Femenino & 2980 & $(48,7)$ \\
\hline \multicolumn{3}{|l|}{ Edad del niño (meses) } \\
\hline 0 a 23 & 2741 & $(44,8)$ \\
\hline 24 a 59 & 3311 & $(54,1)$ \\
\hline Sin datos & 69 & $(1,1)$ \\
\hline \multicolumn{3}{|l|}{ Orden de nacimiento } \\
\hline Primero & 2053 & $(33,5)$ \\
\hline Segundo & 1826 & $(29,8)$ \\
\hline Tercero a más & 2242 & $(36,6)$ \\
\hline \multicolumn{3}{|l|}{ Edad materna } \\
\hline 15 a 24 & 1617 & $(26,4)$ \\
\hline 25 a 34 & 2847 & $(46,5)$ \\
\hline 35 a 49 & 1657 & $(27,1)$ \\
\hline \multicolumn{3}{|l|}{ Educación materna } \\
\hline Sin educación / primaria & 1519 & $(24,8)$ \\
\hline Secundaria & 2983 & $(48,7)$ \\
\hline Superior & 1620 & $(26,5)$ \\
\hline \multicolumn{3}{|c|}{ Índice de masa corporal materno } \\
\hline Ni sobrepeso ni obesidad & 2268 & $(37,0)$ \\
\hline Sobrepeso & 2506 & $(40,9)$ \\
\hline Obesidad & 1348 & $(22,0)$ \\
\hline \multicolumn{3}{|l|}{ Talla materna (metros) } \\
\hline$<1,50$ & 2280 & $(37,2)$ \\
\hline $1,50 a<1,55$ & 2135 & $(34,9)$ \\
\hline$\geq 1,55$ & 1706 & $(27,9)$ \\
\hline \multicolumn{3}{|l|}{ Región natural } \\
\hline Lima Metropolitana & 1733 & $(28,3)$ \\
\hline Resto de costa & 1638 & $(26,8)$ \\
\hline Sierra & 1820 & $(29,7)$ \\
\hline Selva & 931 & $(15,2)$ \\
\hline \multicolumn{3}{|l|}{ Zona de residencia } \\
\hline Urbana & 4441 & $(72,6)$ \\
\hline Rural & 1680 & $(27,4)$ \\
\hline \multicolumn{3}{|l|}{ Metros de altitud } \\
\hline$<2500$ & 4734 & $(77,3)$ \\
\hline$\geq 2500$ & 1388 & $(22,7)$ \\
\hline \multicolumn{3}{|l|}{ Índice de riqueza } \\
\hline Muy pobre & 1218 & $(19,9)$ \\
\hline Pobre & 1474 & $(24,1)$ \\
\hline No pobre & 3429 & $(56,0)$ \\
\hline \multicolumn{3}{|l|}{ Tipo de parto } \\
\hline Vaginal & 4461 & $(72,9)$ \\
\hline Cesárea & 1660 & $(27,1)$ \\
\hline \multicolumn{3}{|l|}{ Complicaciones durante el parto } \\
\hline No & 4648 & $(75,9)$ \\
\hline Sí & 1474 & $(24,1)$ \\
\hline \multicolumn{3}{|l|}{ Complicaciones posparto } \\
\hline No & 3997 & $(65,3)$ \\
\hline Sí & 2125 & $(34,7)$ \\
\hline \multicolumn{3}{|l|}{ Peso al nacer } \\
\hline Bajo & 415 & $(6,8)$ \\
\hline Normal & 5380 & $(87,9)$ \\
\hline Macrosomía & 326 & $(5,3)$ \\
\hline \multicolumn{3}{|l|}{ Obtención del peso al nacer } \\
\hline Tarjeta de control & 2755 & $(45,0)$ \\
\hline Recuerdo de la madre & 3367 & $(55,0)$ \\
\hline
\end{tabular}


Tabla 2. Asociación entre macrosomía y variables seleccionadas: resultados del análisis bivariado*

\begin{tabular}{|c|c|c|c|}
\hline Variable & $\begin{array}{c}\text { Macrosomía } \\
(\%)\end{array}$ & OR (IC 95\%) & valor $p$ \\
\hline \multicolumn{4}{|l|}{ Sexo } \\
\hline Masculino & 7,6 & 1 & $<0,001$ \\
\hline Femenino & 2,4 & $0,29(0,19-0,45)$ & \\
\hline \multicolumn{4}{|l|}{$\begin{array}{l}\text { Orden de } \\
\text { nacimiento }\end{array}$} \\
\hline Primero & 2,0 & 1 & $<0,001$ \\
\hline Segundo & 4,3 & $2,18(1,24-3,62)$ & \\
\hline Tercero a más & 8,9 & $4,58(2,76-7,61)$ & \\
\hline \multicolumn{4}{|l|}{ Edad materna } \\
\hline 15 a 24 & 2,2 & 1 & $<0,001$ \\
\hline 25 a 34 & 5,0 & $2,32(1,32-4,06)$ & \\
\hline 35 a 49 & 8,0 & $2,85(2,18-6,80)$ & \\
\hline \multicolumn{4}{|l|}{ Educación materna } \\
\hline $\begin{array}{l}\text { Sin educación / } \\
\text { primaria }\end{array}$ & 3,4 & 1 & $<0,088$ \\
\hline Secundaria & 5,4 & $1,63(0,99-2,71)$ & \\
\hline Superior & 5,9 & $1,78(1,03-3,07)$ & \\
\hline \multicolumn{4}{|l|}{$\begin{array}{l}\text { Índice de masa } \\
\text { corporal materno }\end{array}$} \\
\hline $\begin{array}{l}\text { Ni sobrepeso ni } \\
\text { obesidad }\end{array}$ & 2,8 & 1 & $<0,001$ \\
\hline Sobrepeso & 5,8 & $2,19(1,39-3,45)$ & \\
\hline Obesidad & 7,8 & $2,99(1,84-4,88)$ & \\
\hline
\end{tabular}

Talla materna

(metros)

$\begin{array}{lcc}<1,50 & 2,0 & 1\end{array}<0,001$

Región natural

\begin{tabular}{|c|c|c|c|}
\hline $\begin{array}{l}\text { Lima } \\
\text { Metropolitana }\end{array}$ & 7,7 & 1 & $<0,001$ \\
\hline Resto de costa & 4,6 & $0,58(0,36-0,92)$ & \\
\hline Sierra & 3,2 & $0,40(0,26-0,64)$ & \\
\hline Selva & 3,9 & $0,51(0,29-0,90)$ & \\
\hline \multicolumn{4}{|l|}{ Zona de residencia } \\
\hline Urbana & 5,6 & 1 & $<0,038$ \\
\hline Rural & 3,6 & $0,64(0,41-0,99)$ & \\
\hline
\end{tabular}

Metros de altitud

\begin{tabular}{cccc}
$<2500$ & 5,9 & 1 & $<0,001$ \\
$\geq 2500$ & 2,2 & $0,34(0,19-0,61)$ & \\
Índice de riqueza & & \multicolumn{2}{c}{$<0,001$} \\
Muy pobre & 3,1 & 1 & $1,03(0,52-2,03)$ \\
Pobre & 3,3 & $2,08(1,20-3,60)$ & \\
\hline No pobre & 6,3 & &
\end{tabular}

*Análisis realizado solo con los participantes para los cuales el peso al nacer se obtuvo de la tarjeta de control.
Tabla 3. Asociación entre macrosomía y variables seleccionadas: resultados del análisis multivariado*

\begin{tabular}{|c|c|c|}
\hline Variable & OR & (IC 95\%) \\
\hline \multicolumn{3}{|l|}{ Sexo } \\
\hline Masculino & 1 & \\
\hline Femenino & 0,28 & $(0,18-0,42)$ \\
\hline \multicolumn{3}{|l|}{ Orden de nacimiento } \\
\hline Primero & 1 & \\
\hline Segundo & 2,18 & $(1,19-2,40)$ \\
\hline Tercero o más & 5,38 & $(2,85-10,16)$ \\
\hline \multicolumn{3}{|l|}{ Edad materna } \\
\hline 15 a 24 & 1 & \\
\hline 25 a 34 & 0,99 & $(0,52-1,87)$ \\
\hline 35 a 49 & 1,09 & $(0,54-2,20)$ \\
\hline \multicolumn{3}{|l|}{ Educación materna } \\
\hline Sin educación / primaria & 1 & \\
\hline Secundaria & 1,37 & $(0,74-2,54)$ \\
\hline Superior & 1,53 & $(0,74-3,15)$ \\
\hline \multicolumn{3}{|c|}{ Índice de masa corporal materno } \\
\hline Ni sobrepeso ni obesidad & 1 & \\
\hline Sobrepeso & 1,52 & $(0,93-2,48)$ \\
\hline Obesidad & 2,08 & $(1,22-3,54)$ \\
\hline \multicolumn{3}{|l|}{ Talla materna (metros) } \\
\hline$<1,50$ & 1 & \\
\hline $1,50 \mathrm{a}<1,55$ & 2,15 & $(1,22-3,78)$ \\
\hline$\geq 1,55$ & 5,07 & $(2,98-8,64)$ \\
\hline
\end{tabular}

Región natural

$\begin{array}{lrr}\text { Lima Metropolitana } & 1 & \\ \text { Resto de costa } & 0,56 & (0,34-0,93) \\ \text { Sierra } & 0,78 & (0,40-1,52) \\ \text { Selva } & 0,50 & (0,25-0,98)\end{array}$

Zona de residencia

$\begin{array}{lrr}\text { Urbana } & 1 & \\ \text { Rural } & 1,48 & (0,83-1,82)\end{array}$

\section{Metros de altitud}

\begin{tabular}{|c|c|c|}
\hline$<2500$ & 1 & \\
\hline$\geq 2500$ & 0,38 & $(0,17-0,87)$ \\
\hline
\end{tabular}

\begin{tabular}{lrr} 
Índice de riqueza & & \\
Muy pobre & 1 & \\
\hline Pobre & 0,88 & $(0,40-1,94)$ \\
\hline No pobre & 1,10 & $(0,44-2,71)$ \\
\hline
\end{tabular}

Análisis realizado solo con los participantes para los cuales el peso al nacer se obtuvo de la tarjeta de control. 
Tabla 4. Frecuencia de complicaciones y tipo de parto de acuerdo al peso al nacer*

\begin{tabular}{|c|c|c|c|c|}
\hline & Frecuencia (\%) & OR & (IC 95\%) & Valor $p$ \\
\hline \multicolumn{5}{|c|}{ Complicaciones durante el parto } \\
\hline Normal & 25,3 & 1 & & 0,480 \\
\hline Macrosomía & 28,0 & 1,15 & $(0,78-1,70)$ & \\
\hline \multicolumn{5}{|c|}{ Complicaciones posparto } \\
\hline Normal & 35,7 & 1 & & 0,460 \\
\hline Macrosomía & 32,6 & 0,87 & $(0,60-1,27)$ & \\
\hline \multicolumn{5}{|l|}{ Cesárea } \\
\hline Normal & 26,9 & 1 & & $<0,001$ \\
\hline Macrosomía & 43,9 & 2,13 & $(1,50-3,04)$ & \\
\hline
\end{tabular}

*Análisis realizado solo con los participantes para los cuales el peso al nacer se obtuvo de la tarjeta de control

\section{DISCUSIÓN}

La prevalencia de macrosomía en el Perú fue relativamente baja $(5,3 \%)$ en comparación con la de otros estudios ${ }^{(6,7)}$. Fue más frecuente en los niños de sexo masculino, cuando el orden de nacimiento fue mayor (tercer hijo o más), en los niños nacidos de madres más altas, madres obesas, en zonas geográficas de baja altitud y en Lima Metropolitana. La macrosomía no se asoció con complicaciones, ya sea durante el parto o después del parto. Sin embargo, los bebés macrosómicos nacieron más frecuentemente por cesárea. Reducir el exceso de peso corporal y la obesidad en las mujeres en edad reproductiva en el Perú puede disminuir potencialmente la macrosomía y las tasas de cesáreas.

Hay que tener cuidado cuando se comparan las prevalencias de macrosomía, ya que en las investigaciones se pueden utilizar diferentes definiciones. La macrosomía se define normalmente como un peso al nacer mayor del percentil 90, por encima de dos desviaciones estándar para la edad gestacional o superior a $4000-4500 \mathrm{~g}^{(1)}$. La prevalencia de macrosomía varía del 5 al $20 \%$ en los estudios desarrollados en países de ingresos altos ${ }^{(6)}$ y de 0,5 a $14,9 \%$ en los países de bajos ingresos ${ }^{(7)}$. Por lo tanto, la prevalencia observada en este estudio fue baja comparada con la de los países de altos ingresos y en el segundo quintil de los datos de los países de bajos ingresos. Se identificó solo un estudio anterior que informa sobre la prevalencia de macrosomía en el Perú.

Este fue un estudio multicéntrico basado en estadísticas de partos institucionales ${ }^{(7)}$, y se estimó una prevalencia de macrosomía del $6,8 \%$, ligeramente superior a la detectada en nuestro estudio. Si los neonatos no nacidos en establecimientos de salud fueron registrados oficialmente con menos frecuencia, esto podría explicar, en parte, la diferencia observada. La prevalencia en este estudio estaba dentro del límite inferior de la observada en los países de ingresos altos. Por lo tanto, si factores similares están actuando para aumentar la prevalencia de macrosomía en países de altos y bajos ingresos, tales como el aumento de la incidencia de la obesidad y la diabetes en las mujeres, se esperaría que la prevalencia de macrosomía en el Perú fuera a aumentar en el futuro. Sin embargo, se necesitan más estudios para investigar esta hipótesis.

Consistentemente con un estudio anterior ${ }^{(6)}$, hemos encontrado los siguientes factores asociados con macrosomía: sexo masculino del recién nacido, ser el tercer hijo o más, aumento de la talla materna y aumento del peso materno. En nuestro estudio, la edad materna, la educación materna, el índice de riqueza y el área rural-urbana de residencia no se asociaron con macrosomía. Li et al. ${ }^{(13)}$ analizaron más de 100000 recién nacidos a término y observaron que el riesgo de macrosomía se asoció positivamente con la edad materna, el IMC antes del embarazo, la gravidez, paridad, talla materna, el aumento de peso gestacional, la diabetes mellitus gestacional y el sexo masculino del feto. EI IMC materno, la edad gestacional y el aumento de peso gestacional fueron los tres factores de riesgo más fuertemente asociados con macrosomía.

La macrosomía fue más frecuente en Lima Metropolitana, independientemente de otras variables en el modelo. De manera similar a los hallazgos del estudio multicéntrico basado en establecimientos de salud (7), ocurren más nacimientos en establecimientos de salud de Lima en comparación con los de otras regiones, lo que podría explicar, en parte, estos resultados. Vale la pena mencionar que este hallazgo era independiente de la obesidad materna.

En los países de bajos ingresos, el nivel socioeconómico se asocia positivamente con la obesidad en hombres y mujeres; por lo tanto, los individuos más ricos y/o aquellos con educación superior son más propensos a la obesidad ${ }^{(14)}$. Si se espera que la macrosomía 
se incremente si aumenta la obesidad, los países de bajos ingresos pueden sufrir consecuencias más drásticas, ya que a menudo tienen menos recursos para prevenir o tratar las complicaciones de la macrosomía, incluyendo las cesáreas. Por lo tanto, la disminución de la macrosomía puede disminuir las tasas de cesárea.

En Perú, similar a los estudios de otros países, la frecuencia de macrosomía se asoció con variables biológicas, que apoya la idea de que es una condición que se produce de manera indistinta tanto en poblaciones ricas como pobres. Sin embargo, se observó macrosomía con mayor frecuencia en madres obesas. Por lo tanto, puede estar indirectamente relacionada con factores socioeconómicos y culturales, tales como la dieta, debido a que la obesidad está asociada con este tipo de variables ${ }^{(14,15)}$.

La altitud ha sido previamente asociada con resultados perinatales y neonatales adversos en investigaciones llevadas a cabo en Perú ${ }^{(16,17)}$ y, por ello, se incluyó esta variable en nuestro análisis. Sin embargo, no se detectó una asociación significativa de una residencia en zonas de gran altitud con la macrosomía en nuestro modelo final. Específicamente, la macrosomía no se asoció con la residencia en la sierra.

Varias complicaciones para el recién nacido y la madre se han asociado con la macrosomía, incluyendo asfixia perinatal, muerte neonatal, distocia de hombros, trabajo de parto prolongado, hemorragia anormal y traumatismo perineal (18). Sin embargo, no se detectó diferencias significativas en la frecuencia de complicaciones entre los recién nacidos macrosómicos y de peso normal. La frecuencia de cesárea entre los recién nacidos macrosómicos fue mayor, sin embargo, aunque es probable que la macrosomía fue la razón de la cirugía, no podemos confirmar esta hipótesis con nuestros datos. Por otra parte, no pudimos determinar si la cesárea fue electiva o de emergencia. En cualquier caso, debido a que la macrosomía se asoció con la obesidad materna, como se ha reportado previamente ${ }^{(19,20)}$, la reducción de la obesidad puede reducir la macrosomía, disminuyendo así las complicaciones en el parto y el puerperio y las tasas de cesáreas. Además, la reducción de la macrosomía puede contribuir a prevenir el desarrollo de sobrepeso / obesidad en la infancia ${ }^{(21)}$.

Una limitación de nuestro estudio es la variabilidad en la definición de macrosomía. Nuestro estudio incluyó solo a los individuos con un peso al nacer de $4000 \mathrm{~g} \mathrm{o}$ más. Además, únicamente se estudiaron los niños que estaban vivos. Los niños que murieron al nacer podrían haber sido macrosómicos; sin embargo, ya que esto es relativamente poco común, no creemos que alteraría sustancialmente nuestros resultados. Además, debido al diseño transversal, no hemos podido comprobar los cambios de la frecuencia de macrosomía a lo largo del tiempo. Por otra parte, solo se incluyeron variables sociodemográficas y biológicas. Otras variables, no recogidas en la ENDES, podrían haber sesgado las asociaciones analizadas (como la diabetes mellitus y diabetes gestacional). En nuestro análisis se utilizaron datos de un estudio basado en la población; por lo tanto, solo se analizaron los pesos al nacer, registrados en las tarjetas de control para obtener datos más fiables. La mayoría de los estudios previos de los países en desarrollo no estaban basados en la población o se utilizan muestras pequeñas. Además, Perú, con regiones geográficas diferentes, proporciona una oportunidad singular para estudiar las variaciones en la macrosomía.

En conclusión, se observó que la prevalencia de macrosomía en el Perú fue relativamente baja $(5,3 \%)$ en comparación con la observada en otros estudios, en particular los de los países de altos ingresos. La macrosomía se observó con mayor frecuencia en niños varones de orden de nacimiento posteriores (tercer hijo o más) y en los niños nacidos de madres obesas, más altas, que viven en altitudes bajas y en Lima Metropolitana. La macrosomía no se asoció con complicaciones en el parto, pero las cesáreas fueron más frecuentes en recién nacidos macrosómicos. Reducir el exceso de peso y la prevención de la obesidad en las mujeres en edad reproductiva en el Perú puede disminuir potencialmente la macrosomía y las tasas de cesáreas.

Agradecimientos: agradecemos el apoyo de la Universidad Internacional de Andalucía (España) en el contexto de la Maestría Internacional de Salud Infantil Comunitaria. Este programa, desarrollado en conjunto con la Universidad Nacional Mayor San Marcos, ha facilitado esta investigación.

Conflictos de interés: los autores declaramos no tener conflictos de interés.

Fuente de financiamiento: autofinanciado.

Contribuciones de los autores: AJL, MS, CG y JA han participado en la concepción del artículo. AJL, y CG participaron del análisis e interpretación de datos. AJL participó en la redacción del artículo. MS, CG y JA revisaron críticamente el artículo. Todos los autores aprobaron la versión final.

\section{REFERENCIAS BIBLIOGRÁFICAS}

1. Boulet SL, Alexander GR, Salihu HM, Pass M. Macrosomic births in the united states. Determinants, outcomes, and proposed grades of risk. Am J Obstet Gynecol. 2003;188(5):1372-8.
2. Grassi AE, Giuliano MA. The neonate with macrosomia. Clin Obstet Gynecol. 2000;43(2):340-8.

3. Kim SY, Sharma AJ, Sappenfiled W, Wilson HG, Salihu HM. Association of maternal body mass index, excessive weight gain, and gestacional diabetes mellitus with large-for-gestational-age births. Obstet Gynecol. 014 Apr;123(4):737-44. doi: 10.1097/AOG.0000000000000177. 
4. Chauhan SP, West DJ, Scardo JA, Boyd JM, Joiner J, Hendrix NW. Antepartum detection of macrosomic fetus: clinical versus sonographic, including soft-tissue measurements. Obstet Gynecol. 2000;95(5):639-42.

5. Haram K, Pirhonen J, Bergsjo P. Suspected big baby: a difficult clinical problem in obstetrics. Acta Obstet Gynecol Scand. 2002;81(3):185-94.

6. Henriksen T. The macrosomic fetus: a challenge in current obstetrics. Acta Obstet Gynecol Scand. 2008;87(2):134-45. doi: 10.1080/00016340801899289.

7. Koyanagi A, Zhang J, Dagvadorj A, Hirayama F, Shibuya K, Souza JP, et al. Macrosomia in 23 developing countries: an analysis of a multicountry, facilitybased, cross-sectional survey. Lancet. 2013 Feb 9;381(9865):476-83. doi: $10.1016 / \mathrm{S} 0140-6736(12) 61605-5$.

8. Chatfield J. ACOG issues guidelines on fetal macrosomia. American College of Obstetricians and Gynecologists. Am Fam Physician. 2001;64(1):169-70.

9. Walsh JM, McAuliffe FM. Prediction and prevention of the macrosomic fetus. Eur J Obstet Gynecol Repro Biol. 2012 Jun;162(2):125-30. doi: 10.1016/j.ejogrb.2012.03.005.

10. Instituto Nacional de Estadística e Informática Perú (INEI). Ficha técnica: Encuesta demográfica y de salud familiar- ENDES 2013. Lima: INEI; 2013.

11. Rutstein S, Kiertsten J. The DHS Wealth Index. DHS comparative
Reports No 6 [Internet]. Calverton, Maryland: ORD Macro. 2014 [cited 2015 Mar 10]. Available from: https:// dhsprogram.com/pubs/pdf/CR6/ CR6.pdf

12. Rutstein S, Rojas G. Guide to DHS statistic Demographic and health surveys methodology [Internet]. [cited 2014 Feb 27]. Available from: http://www.ophi.org.uk/wp-content/ uploads/OPHI-HDCA-SS2012. Guide_DHS_Statistics.pdf

13. LI G, Kong L, Li Z, Zhang L, Fan $\mathrm{L}$, Chen $\mathrm{Y}$, et al. Prevalence of macrosomia and its risk factor in china: a multicenter survey based on birth data involving 101,723 singleton terms infants. Paediatr Perinat Epidemiol. 2014 Jul;28(4):345-50. doi: 10.1111/ ppe.12133.

14. Dinsa G, Goryakin Y, Fumagalli E, Suhrcke M. Obesity and socioeconomic status in developing countries: a systematic review. Obes Rev Obes Rev. 2012 Nov;13(11):1067-79. doi: 10.1111/j.1467-789X.2012.01017.x.

15. García-Fernández E, Rico-Cabanas L, Rosgaard N, Estruch R, BachFaig A. Mediterranean Diet and Cardiodiabesity: A Review. Nutrients. 2014;6:3474-500.

16. Gonzales G, Tapia V, Gasco M, Carrillo C. Maternal hemoglobin concentration and adverse pregnancy outcomes at low and moderate altitudes in Peru. J Matern Fetal Neonatal Med. 2012 Jul;25(7):1105-10. doi: 10.3109/14767058.2011.623200.
17. Gonzales G. Impacto de la altura en el embarazo y en el producto de la gestación. Rev Peru Med Exp Salud Publica. 2012;29(2):242-9.

18. Rossi AC, Mullin P, Prefumo F. Prevention, management, and outcomes of macrosomia: a systematic review of literature and metaanalysis. Obstet Gynecol Surv. . 2013 Oct;68(10):702-9. doi: 10.1097/01. ogx.0000435370.74455.a8.

19. Gaudet L, Wen S, Walker M. The combinated ffect of maternal obesity and fetal macrosomia on pregnancy outcomes. J Obstet Gynaecol Can. 2014;36:776-84.

20. Kamanu C, Onwere S, Chigbu B, Aluka C, Okoro O, Obasi M. Fetal macrosomia in African women: a study of 249 cases. Arch Gynecol Obstet. 2009 Jun;279(6):857-61.

21. Sparano S, Ahrens W, De Henauw S, Marild S, Molnar D, Moreno L, et al. Being macrosomic at birth is an independent predictor of overweight in children: results from the IDEFICS study. Matern Child Health J. 2013 Oct;17(8):1373-81. doi: 10.1007/ s10995-012-1136-2.

Correspondencia: Antonio José Ledo Alves da Cunba

Dirección: Departamento de Pediatria da Faculdade de Medicina da Universidade Federal do Rio de Janeiro

Correoelectrónico:acunha@ufrj.br

\section{Nuestros artículos se encuentran indizados en:}

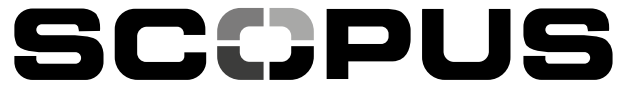

